\title{
A Description of Oral and Swallowing Characteristics in Pediatric Patients with Neuromuscular Diseases
}

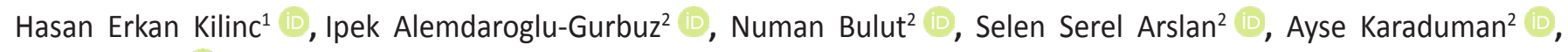 \\ Oznur Yilmaz ${ }^{2}$ \\ ${ }^{1}$ Lokman Hekim University, Faculty of Health Sciences, Physiotherapy and Rehabilitation Department, Ankara, Turkey. \\ ${ }^{2}$ Hacettepe University, Faculty of Physical Therapy and Rehabilitation, Ankara, Turkey.
}

Correspondence Author: Hasan Erkan Kilinc

E-mail: erkankilinc86@hotmail.com

Received: $17.01 .2020 \quad$ Accepted: 17.08 .2020

\begin{abstract}
Objective: The aim of our study was to put forth the existing problems about oral structure/function and swallowing function in patients with different pediatric neuromuscular diseases.

Methods: Forty-five pediatric patients with NMD's aged 10.22 \pm 3.32 years were included in the study. Oral intake levels of patients were determined with Functional Oral Intake Scale. Oral structural and functional examination, assessment of complaints related to swallowing problems, and three-ounce water swallow test were performed to all patients.

Results: In oral assessment, structural problems were determined at varying rates between $13.3 \%$ and $46.6 \%$, problems related to oral functions between $\% 4.4$ and $26.6 \%$, and complaints related to swallowing problems between $11.1 \%$ to $33.3 \%$. Five patients (\%11) failed the three-ounce water swallow test.

Conclusion: The findings related to oral structure/function and swallowing function indicate a risk of swallowing dysfunction with different symptoms and problems in neuromuscular diseases. These symptoms may result in life-threatening complications added to their current neuromuscular problems.
\end{abstract}

Keywords: Deglutition, oral examination, aspiration pneumonia

\section{INTRODUCTION}

Swallowing function consists of a series of sequential movements which provide food passage from oral cavity to stomach. Swallowing problems may occur in any part of oral, pharyngeal and esophageal phases. Swallowing problems which occur in oral phase are related to insufficient bolus control in mouth, inability to turn the food into bolus and, and transport the bolus from mouth to pharyngeal area. The most common problem in pharyngeal phase is inadequate airway protection during swallowing which may lead to lifethreatening problems such as aspiration, pneumonia, and airway obstruction. The problems encountered in esophageal phase are difficulty in transporting the bolus through the esophagus and gastroesophageal reflux_(1).

Dysphagia symptoms were reported in 34.9\%-80\% of patients with pediatric neuromuscular diseases (NMDs) (24). In addition, incidence of aspiration pneumonia increases up to $75 \%$ in this group $(5,6)$.

Piecemeal deglutition, residues in vallecula and pyriform sinuses after semi-solid food swallowing, difficulty in swallowing and mastication problems were seen in patients with spinal muscular atrophy (SMA) (7). Both oral (mastication problems) and pharyngeal phase problems (coughing during feeding, difficulty in swallowing, need to clear throat) were determined in patients with Duchenne muscular dystrophy (DMD) $(8,9)$.

Published studies about swallowing problems in pediatric neuromuscular diseases were found to be performed on subgroups of NMDs. However no consensus on oro-motor and swallowing problems exists in pediatric NMDs (10-12). Thus the aim of our study was to put forth the descriptive characteristics of oral and swallowing problems in a group of pediatric NMD patients with different diagnosis.

\section{METHODS}

\subsection{Participants}

This study was approved by the Non-Invasive Clinical Research Ethics Committee of Hacettepe University (No: GO 16/12528). Written consent forms were signed by all patients and caregivers included in the study. 
Forty-five patients who were diagnosed with one of the neuromuscular disorder by a neurologist were included in the study. Inclusion criteria were as follows: (i) age between 5-18 years, (ii) no additional disease/oral appliance/medicine which may effect oral structures and swallowing functions, (iii) adequate cognitive level to understand and follow instructions for assessments, (iii) full oral intake without restriction.

\subsection{Measures}

Demographic data including age (years), weight $(\mathrm{kg})$, height (cm), and diagnosis were recorded. Oral intake levels of patients were determined with Functional Oral Intake Scale (FOIS) which has adequate reliability, validity, and sensitivity to changes in functional oral intake. FOIS is a scoring system ranging from 1 to 7 for oral intake level of patients ( 1 = nothing by mouth; $7=$ total oral diet with no restrictions) (13). The following assessments were performed to the patients who scored 7 points from the FOIS;

\subsubsection{Oral structural examination}

Oral structural examination was performed with a tongue depressor and an illuminator. Structural characteristics of lip, tongue, jaw, and palate and oral hygiene were assessed during this examination.

The existence of asymmetrical nasolabial folds and/or any disintegrity around labial region at rest were considered as an impairment in lip structure. Abnormally large (macroglossia) or small (microglossia) tongue, short frenulum and disintegrity in tongue were considered as an impairment in tongue structure (14). In the assessment of jaw structure, the alignment of teeth on mandible and maxillary bone with each other was evaluated in anterior and lateral aspects. In anterior aspect, first incisor teeth on maxilla must cover $1 / 2$ portion of first incisor teeth on mandible. The increased rate for this covering was considered closed bite, while decreased rate was considered open bite. In lateral aspect, the first molar teeth on mandible must be placed a little bit anterior to the first molar teeth on maxilla for normal alignment. The patient was determined to have a protruded jaw if the position of first molar teeth on maxilla was placed highly anterior to the first molar teeth on mandible, while he/ she was determined to have a retruded jaw when the first molar teeth on maxilla was placed posterior to the first molar teeth on mandible. These problems were considered as malocclusion (15). In palatal structure evaluation, unusually high and narrow palate is defined as high arched palate by observation and palpation.

\subsubsection{Assessment of oral functions}

The movements intended to be tested were initially performed by the swallowing therapist, then patients were asked to follow these commands.

The commands for the evaluation of tongue functions were to round lips, draw corners back, close lips, puff cheeks and bite lower lip while tip up, tip down, tip right, tip left and tip drawn back along hard palate for lip functions (14). Patients who succeeded these comments were considered to have 'no problem', and those who failed were considered to have 'problem'.

In the supine position the patient was also asked to open their mouths at a maximum level and the distance between the upper and lower teeth incisor teeth was measured with a caliper. Thus, a distance between the upper and lower incisors below $35 \mathrm{~mm}$ was considered as restricted opening of mouth (16).

In addition, the presence of packing food in mouth, leaking food from mouth during feeding, fatigue in the muscles of mastication, fasciculations in tongue, and uncontrolled saliva were asked to patients and/or their caregivers. The answers were recorded as yes or no.

\subsubsection{Assessment of complaints related to swallowing problems}

Presence of coughing, difficulty in swallowing, food getting stuck in throat during feeding, and change in voice quality after meals were assessed by asking to patients and/or caregivers. The frequency of recurrent pulmonary infections in the previous year, persistent wheezing and secretion were also asked.

\subsubsection{Three-ounce water swallow test}

The three-ounce water swallow test is a widely used method of screening individuals who are at risk for oropharyngeal dysphagia and aspiration. Criteria for referral for further assessment of swallowing include inability to complete the task, coughing, choking, or a wet-hoarse vocal quality exhibited either during or within 1 minute of test completion. A swallowing therapist administered the three-ounce water swallow test while the child was placed in a sitting position on a chair with the head upright and midline position and the arms and legs supported. Each patient was given three ounces of water and asked to drink from a cup or straw without interruption, and results were noted (17). Threeounce water swallow test can be used in children between the ages of 2-18 (18).

All evaluations were performed by the same therapist.

\subsection{Data Analysis}

Statistical analysis was performed by using IBM-SPSS for Windows version 20. Descriptive statistics were presented as number/percent $(\mathrm{n} / \%)$ for qualitative data and mean $(\mathrm{X})$ and standard deviation (SD) for quantitative variables.

\section{RESULTS}

The demographic characteristics of the 45 patients included in the study were as given in Table 1. All patients were in FOIS level 7 which means total oral diet with no restrictions $(n=45$, $100 \%)$. 
Table 1. Demographic Characteristics of the Patients.

\begin{tabular}{|lc|}
\hline Demographic characteristics & $\mathbf{X} \pm$ SD \\
\hline Age (years) & $10.22 \pm 3.32$ \\
\hline Height $(\mathrm{cm})$ & $125.42 \pm 16.48$ \\
\hline Weight(kg) & $29.83 \pm 12.42$ \\
\hline
\end{tabular}

X: Mean, SD: Standard Deviation

The diagnostic distribution of the patients was as shown in Table 2.

Table 2. Diagnosis of the Patients.

\begin{tabular}{|lcc|}
\hline Diagnosis & $\mathbf{n}$ & \% \\
\hline Duchenne muscular dystrophy & 25 & 55.6 \\
\hline Becker muscular dystrophy & 1 & 2.2 \\
\hline Spinal muscular atrophy type 2 & 8 & 17.8 \\
\hline Spinal muscular atrophy type 3 & 2 & 4.4 \\
\hline Congenital muscular dystrophy & 5 & 11.1 \\
\hline Guillain Barre syndrome & 1 & 2.2 \\
\hline Mitochondrial myopathy & 2 & 4.4 \\
\hline Dermatomyositis & 1 & 2.2 \\
\hline Total & 45 & 100 \\
\hline
\end{tabular}

\subsection{Results Related to Oral Structural Examination}

No structural abnormality in lips was detected. None of the patients were determined to have foul breath, infection, or tooth decay related to bad oral hygiene. The frequencies of encountered problems in oral structures were as given in Table 3.

Table 3. Oral Structural Abnormalities and Their Frequencies.

\begin{tabular}{|lcc|}
\hline Oral Structural Abnormalities & $\mathbf{n}$ & $\%$ \\
\hline Macroglossia (tongue) & 6 & 13.3 \\
\hline Malocclusion (jaw) & 21 & 46.6 \\
\hline High arched palate & 6 & 13.3 \\
\hline
\end{tabular}

\subsection{Results Related to Assessment of Oral Functions}

All of the patients had normal lip and tongue functions. Packing food in mouth, leaking food from mouth during feeding, and uncontrolled saliva were not seen in oral function assessment. The existing abnormalities in the assessment of oral functions were as given in Table 4.

Table 4. Impairments in Oral Functions.

\begin{tabular}{|lcc|}
\hline Oral Functions & $\mathbf{n}$ & $\%$ \\
\hline Restricted opening of mouth & 2 & 4.4 \\
\hline Fatigue during mastication & 12 & 26.6 \\
\hline Fasciculations in tongue & 4 & 8.8 \\
\hline
\end{tabular}

\subsection{Results of the Assessment of Swallowing Problems}

Complaints related to swallowing problems were as given in Table 5.
Table 5. Swallowing Problems in the Study Population.

\begin{tabular}{|lcc|}
\hline Complaints related to swallowing problems & $\mathbf{n}$ & $\%$ \\
\hline Coughing during feeding & 9 & 20 \\
\hline Difficulty in swallowing & 9 & 20 \\
\hline Food getting stuck in throat during feeding & 8 & 17.7 \\
\hline Change in voice quality after feeding & 5 & 11.1 \\
\hline Recurrent pulmonary infections & 5 & 11.1 \\
\hline Persistent wheezing and secretion & 15 & 33.3 \\
\hline
\end{tabular}

\subsection{Results of the Three-Ounce Water Swallow Test}

Five $(11.1 \%)$ of the patients (2 SMA type 2, 2 CMD, and 1 mitochondrial myopathy) failed the three-ounce water swallow test (Table 6).

Table 6. Three-Ounce Water Swallowing Test.

\begin{tabular}{|lll|}
\hline Diagnosis & $\mathrm{n}$ & $\%$ \\
\hline Duchenne muscular dystrophy & - & - \\
\hline Becker muscular dystrophy & - & - \\
\hline Spinal muscular atrophy type 2 & 2 & 4.4 \\
\hline Spinal muscular atrophy type 3 & - & - \\
\hline Congenital muscular dystrophy & 2 & 4.4 \\
\hline Guillain Barre syndrome & - & - \\
\hline Mitochondrial myopathy & 1 & 2.2 \\
\hline Dermatomyositis & - & - \\
\hline Total & 5 & 11.1 \\
\hline
\end{tabular}

\subsection{The Most Frequent Problems Seen in NMD Subgroups}

Malocclusion ( $n=16,64.0 \%$ ) was found to be the most frequent problem in DMD, while recurrent pulmonary infection and persistent wheezing and secretion $(n=5,62.5 \%)$ was the most common one in SMA type 2. Besides, the patients $(n=2)$ who had restriction in opening of the mouth and the majority $(n=3,75.0 \%)$ of patients who had fasciculation in tongue were diagnosed as SMA type 2. Persistent wheezing and secretion, coughing during feeding, and change in voice quality after feeding were encountered frequently in CMD (3 of the 5 patients, $60.0 \%$ ).

\section{DISCUSSION}

The oral and swallowing characteristics of a group of pediatric patients with different NMD diagnosis were assessed in this study. The intraoral structural impairments in NMDs were macroglossia, malocclusion and high arched palate. Restricted opening of mouth, fatigue during mastication and fasciculations in tongue were found as oral functioning problems. The complaints of patients related to pharyngeal swallowing problems were persistent wheezing and secretion, coughing during feeding, difficulty in swallowing, food getting stuck in throat, change in voice quality after feeding and recurrent pulmonary infection. Also five of the patients failed the three-ounce water swallow test.

Intact oral structures play a crucial role in oral preparation phase in NMDs. Occlusal impairments may lead to problems 
in oral preparatory phase of swallowing function especially in chewing process. Occlusal problems such as open bite may cause problems like leaking food from mouth during feeding and uncontrolled saliva. However, the most important handicap of occlusal impairments is the failure in mechanical breakdown of food for forming appropriate bolus ready to swallow. If foods could not have been converted into a bolus ready for swallowing, appropriate pharyngeal triggering would not occur. As a result of this, all phases of the swallowing would be affected (19). In our study, malocclusion was reported to be the most common oral structural impairment especially in DMD which may lead insufficient chewing function, and a limited number of study reported this problem as one of the predisposing factors for this insufficiency (20).

The tongue should be capable of manipulating the foods sufficiently to convert into bolus. When the tongue becomes extremely hypertrophic (macroglossia) as seen in our study population, it covers a lot of space in the mouth. Hence, this problem does not allow enough mobility of tongue in the mouth. It is emphasized that macroglossia which can be seen in pediatric NMDs may lead to swallowing problems (4). In a videofluroscopic study with 102 DMD patients, reduced tongue mobilization due to macroglossia was reported to lead inefficient bolus transport to the pharyngeal region (21). Tongue fasciculation which is an another impairment seen in our SMA type 2 patients was identified as a common problem in diseases with bulbar weakness previously (22). Basically, fasciculation indicates weak tongue muscles. Thus, loss of muscular strength of the tongue affects swallowing function negatively, rather than fasciculations. Weakened tongue leads to inefficient bolus formation and bolus transporting from base of the tongue to pharyngeal region (23).

High arched palate was commonly reported to be seen in patients with cerebral palsy, and partially in pediatric neuromuscular diseases (24). High arched palate was found to be a problem in our study similar to previous studies. This finding is important for it may cause a disadvantage in transportation viscous and solid foods to the pharynx due to the excessive space between the tongue and the palate (25). This may be the reason of why we had patients with problems with difficulty in swallowing in our study.

Restriction in the opening of the mouth occurs based on different problems such as stiffness in masticator muscles, temporomandibular joint problems, weakness in masticator muscles etc. In our study, restriction in the opening of the mouth in our SMA type 2 patients was thought to be originated from weak masticator muscles. This problem may affect oro-motor functions such as chewing, speaking, and swallowing.

Fatigue during mastication seen in our patients may be associated with the loss of strength and endurance of the masticator muscles. In patients who have fatigue during mastication may have a tendency to swallow bolus without adequate chewing. As a result, insufficiently fragmented and softened foods may cause difficulty in swallowing and residue on vallecula, pharyngeal wall, and pyriform sinuses in pharyngeal phase of the swallowing, and also extended feeding time.

The findings such as coughing during feeding, change in voice quality after feeding, difficulty in swallowing, food getting stuck in throat, persistent wheezing and secretion, and recurrent pulmonary infections were thought to be the signs of pharyngeal swallowing disorders in our pediatric patients with NMDs. Coughing during feeding and change in voice quality after feeding indicate food penetration into the vocal cords which are impairments of airway protection mechanism (26). Problems related to the protection of the airway may be seen in a certain extent in patients with pediatric neuromuscular diseases (23). Difficulty in swallowing depends on inadequate tongue base pressure and deficiency in pharyngeal constructor muscle strength (27). Likewise, food getting stuck in throat is related to insufficient squeezing function in the pharyngeal phase of the swallowing (28). Difficulty in swallowing as well as food getting stuck in throat may lead to residue on pharyngeal wall, vallecula and pyriform sinuses. After swallowing remaining residue in these structures constitute a serious risk factor for aspiration. Moreover, these problems may cause a more dramatic result such as airway obstruction. In recent studies, problems due to insufficient pharyngeal muscle strength have been reported especially in SMA type 2 patients $(8,29)$. The risk of aspiration mentioned above may be the cause of persistent wheezing and secretion, and recurrent pulmonary infections except for problems such as immobilization and weak cough force in NMDs.

Three-ounce water swallow test is an important indicator for detecting insufficient airway protection mechanism supporting our previous findings related to complaints of swallowing disorder. This clinical swallowing evaluation of our patient population showed that the percentage of pharyngeal swallowing disorders was $11.1 \%$. However, approximately $40 \%$ of food aspirations are silent (without coughing). Therefore, the consideration to have adequate airway protection mechanism in patients who successfully performed this test may be misleading. The rate as $11.1 \%$ is also an important rate for this problem may result in lifethreatening conditions.

Our limitations are, not using an advanced assessment method such as videofluoroscopy to detect aspiration symptoms and no following period of time. Studies with larger sample size are needed in the future.

\section{CONCLUSION}

As a conclusion, a large variety of problems related to oral structure, function, and swallowing were observed in pediatric NMDs. The severity of these problems had also a wide spectrum. These patients may present with more destructive manifestations when swallowing problems are added to their current neuromuscular problems. Therefore, swallowing problems should not be ignored in these patients 
due to their life-threatening results. Information obtained for the possible swallowing disorders in patients with NMDs with the use of cheap and easily performed evaluation methods play a key role in terms of consulting to advanced tests such as Videofluoroscopy, Fiberoptic Endoscopic Evaluation of Swallowing (FEES) and appropriate treatment options.

\section{REFERENCES}

[1] Logemann JA. The evaluation and treatment of swallowing disorders. Curr Opin Otolaryngol Head Neck Surg 1998;6(6):395-400.

[2] Hill M, Hughes T, Milford C. Treatment for swallowing difficulties (dysphagia) in chronic muscle disease. Cochrane Database Syst Rev 2004(2):CD004303.

[3] Britton D, Karam C, Schindler JS. Swallowing and Secretion Management in Neuromuscular Disease. Clin Chest Med 2018; 39(2):449-457.

[4] Willig TN, Paulus J, Lacau Saint Guily J, Beon C, Navarro J. Swallowing problems in neuromuscular disorders. Arch Phys Med Rehabil 1994; 75(11):1175-1181.

[5] Matsaniotis N, Karpouzas J, Tzortzatou-Vallianou M, Tsagournis E. Aspiration due to difficulty in swallowing. Arch Dis Child. 1971; 46(250):788-790.

[6] Hirsch AW, Monuteaux MC, Fruchtman G, Bachur RG, Neuman MI. Characteristics of Children Hospitalized With Aspiration Pneumonia. Hosp Pediatr 2016; 6(11):659-666.

[7] Chen YS, Shih HH, Chen TH, Kuo CH, Jong YJ. Prevalence and risk factors for feeding and swallowing difficulties in spinal muscular atrophy types II and III. J Pediatr 2012; 160(3):447451 e1.

[8] Van den Engel-Hoek L, Erasmus C, Van Bruggen H, De Swart B, Sie L, Steenks $M$, et al. Dysphagia in spinal muscular atrophy type II More than a bulbar problem? Neurology 2009; 73(21):1787-1791.

[9] Hanayama K, Liu M, Higuchi Y, Fujiwara T, Tsuji T, Hase K, Ishihara T. Dysphagia in patients with Duchenne muscular dystrophy evaluated with a questionnaire and videofluorography. Disabil Rehabil 2008; 30(7):517-522.

[10] Audag N, Goubau C, Toussaint M, Reychler G. Screening and evaluation tools of dysphagia in adults with neuromuscular diseases: a systematic review. Ther Adv Chronic Dis 2019; 10:204.062.2318821622.

[11] Sproule DM, Montes J, Dunaway S, Montgomery M, Battista V, Koenigsberger D, Martens B, Shen W, Punyanitya M, Benton M, Butler H, Caracciolo J, Mercuri E, Finkel R, Darras $B$, De Vivo DC, Kaufmann P. Adiposity is increased among highfunctioning, non-ambulatory patients with spinal muscular atrophy. Neuromuscul Disord 2010; 20(7):448-452.

[12] Marques TB, Neves Jde C, Portes LA, Salge JM, Zanoteli E, Reed UC. Air stacking: effects on pulmonary function in patients with spinal muscular atrophy and in patients with congenital muscular dystrophy. J Bras Pneumol. 2014;40(5):528-534.
[13] Crary MA, Mann GDC, Groher ME. Initial psychometric assessment of a functional oral intake scale for dysphagia in stroke patients. Archives of physical medicine and rehabilitation. 2005;86(8):1516-1520.

[14] Louis S. KO, Ruscello DM. Oral speech mechanism screening examination (OSMSE). Baltimore University Park Press; 1981.

[15] Zemlin WR. Anatomy and physiology. Speech and hearing science. 4 ed. Englewood Cliffs: Pentice-Hall; 1998.

[16] Abou-Atme YS, Chedid N, Melis M, Zawawi KH. Clinical measurement of normal maximum mouth opening in children. Cranio : the journal of craniomandibular practice. 2008;26(3):191-196.

[17] Suiter DM, Leder SB. Clinical utility of the 3-ounce water swallow test. Dysphagia 2008;23(3):244-250.

[18] Suiter DM, Leder SB, Karas DE. The 3-ounce (90-cc) water swallow challenge: a screening test for children with suspected oropharyngeal dysphagia. Otolaryngol Head Neck Surg. 2009;140(2):187-190.

[19] Corbin-Lewis K, Liss J. Clinical anatomy \& physiology of the swallow mechanism: Nelson Education; 2014.

[20] van Bruggen HW, van de Engel-Hoek L, Steenks MH, Bronkhorst EM, Creugers NH, de Groot IJ, Kalaykova SI. Predictive factors for masticatory performance in Duchenne muscular dystrophy. NMD 2014;24(8):684-692.

[21] Nozaki S, Umaki Y, Sugishita S, Tatara K, Adachi K, Shinno S. Videofluorographic assessment of swallowing function in patients with Duchenne muscular dystrophy. Rinsho shinkeigaku= Clin Neurol 2007;47(7):407-412.

[22] Lunn MR, Wang $\mathrm{CH}$. Spinal muscular atrophy. The Lancet 2008;371(9630):2120-2133.

[23] van den Engel-Hoek L, Erasmus CE, van Hulst KC, Arvedson JC, de Groot IJ, de Swart BJ. Children with central and peripheral neurologic disorders have distinguishable patterns of dysphagia on videofluoroscopic swallow study. J Child Neurol 2013:088.307.3813501871.

[24] van den Engel-Hoek L, de Groot IJ, de Swart BJ, Erasmus CE. Feeding and Swallowing Disorders in Pediatric Neuromuscular Diseases: An Overview. J Neuromuscul Dis 2015;2(4):357369.

[25] Hamanaka-Kondoh S, Kondoh J, Tamine K, Hori K, Fujiwara S, Maeda Y, Matsumuta T, Yasui K, Fujimura H, Sakoda S, Ono T. Tongue pressure during swallowing is decreased in patients with Duchenne muscular dystrophy. NMD 2014;24(6):474481.

[26] Corbin-Lewis K, Liss J. Clinical anatomy \& physiology of the swallow mechanism: Nelson Education; 2014. pp 154-172.

[27] Shaker R, Cook IJ, Dodds WJ, Hogan WJ. Pressure-flow dynamics of the oral phase of swallowing. Dysphagia. 1988;3(2):79-84.

[28] Weckmueller J, Easterling C, Arvedson J. Preliminary temporal measurement analysis of normal oropharyngeal swallowing in infants and young children. Dysphagia 2011; 26(2):135-143.

[29] Chen Y-S, Shih H-H, Chen T-H, Kuo C-H, Jong Y-J. Prevalence and risk factors for feeding and swallowing difficulties in spinal muscular atrophy types II and III. J Pediatr. 2012;160(3):447451. e1. 